\title{
Predictive Risk Factors for Recurrence or Metastasis in Papillary Thyroid Cancer
}

\author{
Wan Wook Kim ${ }^{1}$, Jeeyeon Lee ${ }^{1}$, Jin Hyang Jung ${ }^{1}$, Ho Yong Park', Ji Yun Jeong ${ }^{2}$, \\ Ji-Young Park ${ }^{2}$ and Ralph P. Tufano ${ }^{3}$ \\ Department of Surgery, Kyungpook National University, School of Medicine1, Department of Pathology, Kyungpook National \\ University, School of Medicine', Daegu, Korea, Department of Otolaryngology-Head and Neck Surgery, Johns Hopkins University \\ School of Medicine, Baltimore, MD, USA
}

\begin{abstract}
Background and Objectives: This study investigated predictive risk factors for cervical nodal recurrence or metastasis in papillary thyroid carcinoma (PTC). Materials and Methods: From September 2014 to February 2015, a total of 321 PTC patients were enrolled retrospectively. Except for 154 NO patients, the remaining 167 patients were divided into two groups as follows: Group I ( $n=140)$, central lymph node (LN) metastasis ( $p N 1 a)$; Group II $(n=27)$, lateral $L N$ metastasis $(p N 1 b, n=23)$ or $L N$ recurrence $(n=4)$. The patients who had $L N$ metastasis or recurrence underwent selective LN dissection or recurrent LN excision. Results: Central LN metastases were found in $44.0 \%(142 / 321)$ of patients. Two hundred thirty patients (71.7\%) were classified as being at low-risk for LN disease, as evidenced by NO or fewer than five micrometastases. The mean size of central metastatic LNs was $0.37 \pm 0.34 \mathrm{~cm}$. A total of 76 patients $(46.6 \%)$ presented with micrometastasis, and ten (3.1\%) presented with extranodal extension (ENE). The multiple/bilateral cancer, Extrathyroidal extension, size of metastatic LN, ENE, high risk LN disease $(>5$, macrometastasis, $>3.0 \mathrm{~cm})$ and high thyroglobulin were significant risk factors in predicting $L N$ recurrence or lateral LN metastasis $(p<0.05)$ in univariate analysis. Patients with ENE were 10.3 times more at risk for recurrence or metastasis than patients without ENE. Conclusion: We consider the ENE was the most potent risk factors for LN recurrence or lateral LN metastasis in PTC.
\end{abstract}

Key Words: Metastasis, Papillary thyroid cancer, Recurrence, Risk factors

\section{Introduction}

Papillary thyroid cancer (PTC) is the most frequent, favorable, and the 10-year survival rate was reported to be $96.5 \%{ }^{1,2)}$ The most important prognostic factors affecting PTC patient survival are age, sex, tumor size, extrathyroidal extension (ETE), lymph node (LN) metastasis, and distant metastasis. ${ }^{3,4)}$ Among them, LN metastasis is very common in PTC patients, having been reported in up to $90 \%{ }^{5,6)}$ Despite appropriate treatment, LN recurrence occurs in 5 to $28 \%$ of patients. ${ }^{7,8)}$ Some studies have shown that regional LN metastasis did not significantly affect overall survival rates, while others have suggested that LN metastasis reduced recurrence-free survival as well as overall survival rates, and could be an independent risk factor for local recurrence. ${ }^{9,10)}$

Characteristics of the metastasis were not considered in American Joint Committee on Cancer (AJCC) staging. However, clinical outcomes among the same N1a metastases vary depending on characteristics of the metastasis. Recent studies have reported on the number and size of LN metastasis, LN ratio (involved

Received April 16, 2020 / Revised June 23, 2020 / Accepted July 24, 2020

Correspondence: Wan Wook Kim, MD, PhD, Department of Surgery, Kyungpook National University Chilgok Hospital, Kyungpook National University, School of Medicine, 807 Hogukno, Buk-gu, Daegu 41404, Korea

Tel: 82-53-200-2705, Fax:82-53-200-2027, E-mail: kww1324@naver.com 
$L N /$ retrieved LN), and extranodal extension (ENE) as risk factors for metastasis or recurrence. ${ }^{11-13)}$ Lymph node characteristics were reflected in accordance with the American Thyroid Association Guidelines, which classify low-risk LN disease as pNO or less than $5 \mathrm{mi}$ crometastases, high-risk LN disease, as indicated by five or more, macrometastases, and metastatic LN 3 $\mathrm{cm}$ or greater in size. ${ }^{14-16)}$

We supposed that the patients with central LN metastasis and patients with lateral LN metastasis or LN recurrence would naturally have different clinical implications and also different tumorous features. This study investigated the predictive risk factors of lateral $L N$ metastasis or $L N$ recurrence according to characteristics of LN metastasis.

\section{Materials and Methods}

This study enrolled 321 PTC patients who underwent thyroidectomies and central LN dissections (CLND) at Kyungpook National University Chilgok Hospital from September 2014 to February 2015. Endpoints of this study were lateral $L N$ metastasis or $L N$ recurrence. Except for 154 pN0 patients who had no LN metastasis or recurrence among 321 patients, a total of $167 \mathrm{pa}-$ tients were divided into two groups as follows: Group I $(n=140)$, central LN metastasis (pN1a); Group ॥ $(n=27)$, lateral $L N$ metastasis ( $p N 1 b, n=23)$ or $L N$ recurrence in central or lateral compartment $(n=4)$. Recurrence was defined as the occurrence of a LN with cancer cells at the previous surgical site.
The clinicopathologic data of patients were retrospectively collected using patient medical records. All patients underwent LN evaluation with high resolution ultrasonography (US). Extrathyroidal extension included microscopic or gross tumor extension. All patients underwent evaluation for recurrence via neck US and thyroglobulin assessment once every six to 12 months post-operatively. Size of metastatic LN was defined as its size as presented in the largest microscopic metastatic focus. Micrometastasis was defined as deposits of less than $0.2 \mathrm{~cm}$. The LN ratio was defined as the number of metastatic LNs divided by the number of retrieved LNs. Other studies about metastatic $L N$ ratio concluded the appropriate cut-off value was 0.38 from the Receiver Operating Characteristic curve (ROC) analysis; therefore, 0.4 was used as the cut-off for the LN ratio in this study. ${ }^{11)}$ ENE was defined as the extent of tumor invasion of the LN capsule.

Student's t-test analyses were performed to evaluate differences in the arithmetic means of two populations. Categorical variables were analyzed using Pearson's Chi-square and Fisher's exact test. Statistical significance was assessed by logistic regression analysis to predict whether significant risk factors as determined by univariate analysis affected recurrence or lateral LN metastasis. All statistical calculations were conducted and analyzed using SPSS version 20.0 for Windows.

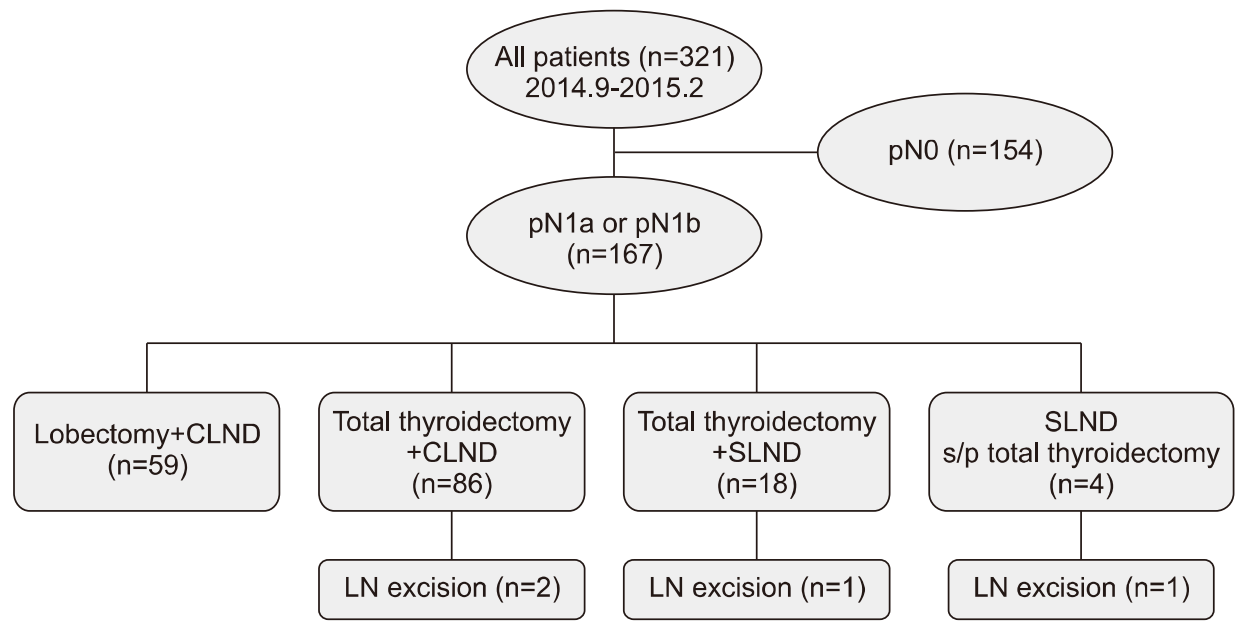

Fig. 1. It shows a flow chart of all 321 patients enrolled. CLND: central lymph node dissections, SLND: selective lymph node dissection 


\section{Results}

All 167 patients received surgeries, with 59 receiving a lobectomy, 86 a total thyroidectomy, 18 a total thyroidectomy \& selective LN dissection (SLND, level $\|-V)$, four SLND during the surveillance period after thyroid surgery (Fig. 1). Among them, three cases of recurred $L N$ excision in the level VI, and one retropharyngeal LN excision. Four SLND were performed on average of 26.2 months after total thyroidectomy and LN excision on 27.5 months after first operation. Therapeutic CLND was performed in 40 cases (12.5\%) and the remaining all patients was treated with prophylactic CLND. Clinicopathological characteristics of patients are shown in Table 1. The mean tumor size was $0.84 \mathrm{~cm}$ (range $0.1-5.0$ ), and papillary thyroid microcarcinoma (PTMC) was $74.1 \%$. Of the 236 cases of unilateral cancer, 15 cases (6.4\%) showed contralateral LN metastasis without ipsilateral LN metastasis. ETE was seen in $56.1 \%$ and lymphovascular invasion in $10.3 \%$. Lymph node micrometastasis in the central compartment was found in 76 cases (46.6\%), and ENE in 10 cases (3.1\%). The mean $L N$ ratio was 0.19 , and 60 cases $(18.7 \%)$ demonstrated a high LN ratio $(>0.4)$. Low-risk for LN disease was found in 230 cases (71.7\%). Radioactive iodine (RAI) treatment was performed in 66 patients (20.5\%). No patients presented with death or distant metastasis during the follow-up period.

Clinicopathologic results were compared by study group according to LN status with pN1 and with recurrence or lateral LN metastasis (Table 2). In univariate analyses, 7 variables were significantly different between these two groups; these were multiple/bilateral cancer, ETE, macrometastasis, ENE, high-risk LN disease and high thyroglobulin. Only those factors that were significant in the univariate test were analyzed with a logistic regression test (Table 3). Extranodal extension (odds ratio [OR], 10.30; $\mathrm{p}=0.021$ ) was only significant risk factor for recurrence or lateral LN metastasis. The mean follow-up period was 61.9 months (range, 28.7-65.8).

Table 1. Demographic and the clinicopathologic characteristics of 321 patients with papillary thyroid cancer

\begin{tabular}{|c|c|}
\hline Variables & Mean $(n=321$, range $)$ \\
\hline Age (years) & $45.3 \pm 10.5(15-78)$ \\
\hline Gender (M:F, \%) & $54: 267(16.8 \%: 83.2 \%)$ \\
\hline Tumor size $(\mathrm{cm})$ & $0.84 \pm 0.59(0.1-5.0)$ \\
\hline${ }^{\dagger}$ Multiple cancer & $127(39.6 \%)$ \\
\hline Bilateral cancer & $85(26.5 \%)$ \\
\hline Extrathyroidal extension & $180(56.1 \%)$ \\
\hline N0:N1a:N1b & $154(48.0 \%): 142(44.0 \%): 25(8.0 \%)$ \\
\hline Retrieved LN (n) & $11.0 \pm 13.6(0-113)$ \\
\hline Central & $8.0 \pm 5.7(0-31)$ \\
\hline Lateral & $40.8 \pm 20.0(16-103)$ \\
\hline Metastatic LN (n) & $1.9 \pm 3.3(0-23)$ \\
\hline Central & $1.6 \pm 2.5(0-17)$ \\
\hline Lateral & $4.4 \pm 4.4(1-18)$ \\
\hline High number of metastatic LN $(\geq 2)$ & $103(32.1 \%)$ \\
\hline \multicolumn{2}{|l|}{ Metastatic LN size $(\mathrm{cm})$} \\
\hline Central & $0.37 \pm 0.34(0.1-2.1)$ \\
\hline Lateral & $0.89 \pm 0.55(0.3-2.7)$ \\
\hline Micrometastasis $(<0.2 \mathrm{~cm})$ at level VI & 76/163 (46.6\%) \\
\hline Extranodal extension at level VI & $10(3.1 \%)$ \\
\hline$L N$ ratio (metastatic $L N /$ retrieved $L N$ ) & $0.19 \pm 0.27(0-1.0)$ \\
\hline High LN ratio $(>0.4)$ & $60(18.7 \%)$ \\
\hline Low risk disease (N0, $\leq 5$ micrometastasis) & $230(71.7 \%)$ \\
\hline
\end{tabular}

${ }^{\dagger}$ Multiple cancer: multiple tumor in unilateral lobe except bilateral tumor 
Risk Factors for Recurrence and Metastasis

Table 2. Clinicopathologic factors of study group according to lymph node status in papillary thyroid cancer

\begin{tabular}{lccc}
\multicolumn{1}{c}{ Variables } & $\begin{array}{c}\text { Group I } \\
(\mathrm{n}=140)\end{array}$ & $\begin{array}{c}\text { Group II } \\
(\mathrm{n}=27)\end{array}$ & p value \\
\hline Age (years) & $43.7 \pm 11.0$ & $44.5 \pm 10.2$ & 0.729 \\
Gender (Male) & $26(18.6 \%)$ & $8(29.6 \%)$ & 0.191 \\
Tumor size (cm) & $0.96 \pm 0.66$ & $1.13 \pm 0.51$ & 0.568 \\
${ }^{*}$ PTMC & $94(67.19 \%)$ & $15(55.6 \%)$ & 0.247 \\
${ }^{\dagger}$ Multiple cancer & $64(45.7 \%)$ & $18(66.7 \%)$ & 0.046 \\
Bilateral cancer & $45(32.1 \%)$ & $16(59.3 \%)$ & 0.007 \\
Extrathyroidal extension & $84(60.0 \%)$ & $26(96.3 \%)$ & 0.000 \\
High number of metastatic LN ( $\geq 2)$ & $85(60.7 \%)$ & $18(66.7 \%)$ & 0.560 \\
Macrometastasis on LN & $66(47.1 \%)$ & $19(70.4 \%)$ & 0.002 \\
Extranodal extension & $4(2.9 \%)$ & $6(22.2 \%)$ & 0.000 \\
LN ratio & $0.38 \pm 0.25$ & $0.34 \pm 0.30$ & 0.225 \\
High LN ratio ( $\geq 0.4)$ & $52(37.1 \%)$ & $11(40.7 \%)$ & 0.724 \\
High-risk LN disease & $71(50.71 \%)$ & $20(74.1 \%)$ & 0.026 \\
Off-hormone Thyroglobulin & $1.33 \pm 2.27$ & $6.69 \pm 17.05$ & 0.002 \\
\hline
\end{tabular}

*PTMC: papillary thyroid microcarcinoma (tumor size $\leq 1.0 \mathrm{~cm}$ )

${ }^{\dagger}$ Multiple cancer: multiple tumor in unilateral lobe except bilateral tumor

Table 3. Multivariate logistic regression for factors in relation to lymph node recurrence or lateral lymph node metastasis

\begin{tabular}{lcccc}
\multicolumn{1}{c}{ Variables } & B & Sig. & Exp(B) & $95 \% \mathrm{Cl}$ \\
\hline${ }^{\dagger}$ Multiple cancer & 0.382 & 0.694 & 1.466 & $0.218-9.875$ \\
Bilateral cancer & 0.790 & 0.398 & 2.204 & $0.353-13.751$ \\
Extrathyroidal extension & 2.458 & 0.097 & 9.410 & $1.418-96.262$ \\
Macrometastasis on LN & -0.205 & 0.870 & 0.814 & $0.070-9.508$ \\
Extranodal extension & 2.491 & 0.021 & 10.309 & $2.343-62.187$ \\
High risk LN disease & 1.537 & 0.256 & 4.649 & $0.328-65.803$ \\
Thyroglobulin & 0.099 & 0.270 & 1.104 & $0.926-1.318$ \\
\hline
\end{tabular}

${ }^{\dagger}$ Multiple cancer: multiple tumor in unilateral lobe except bilateral tumor

B: beta, Sig.: significance, $\operatorname{Exp}(B)$ : exponential (beta)

\section{Discussion}

Although the relationship between LN metastasis and prognosis remains controversial, most studies agree that LN metastasis is an important risk factor for locoregional recurrence and distant metastasis, and complete removal of the metastatic $L N$ is generally performed to reduce the risk for local recurrence. ${ }^{9,10)}$ In large-scale, population-based, nested case-control studies, PTC patients with LN metastasis showed high levels of mortality, so LN metastasis was found to be an important prognostic factor. ${ }^{10)}$ Reasons for contrasting results regarding the impact of $L N$ metastasis on the risk factors for local recurrence are because $L N$ metastasis status was not expanded upon according to various factors, such as metastatic size, number of metastases, and ENE, and risk depending on these factors was not stratified. The accurate evaluation of LN metastasis is important in assessing the risk of locoregional recurrence and establishing treatment strategies.

\section{Number of Metastatic LNs}

Lee et al. ${ }^{17)}$ reported that number of metastatic LN was a prognostic factor in recurrence and that the recurrence-free rate differed significantly between the two or more metastatic LNs group and the zero or one group, with a recurrence rate that was 2.3 times higher in the former, and 3.7 times higher among patients with more than six metastatic LNs. Based on these data, they recommended that the number of meta- 
static LNs should be considered when staging, and patients with two or more metastatic LNs were at a higher risk of recurrence and should be considered for treatment with additional RAI ablation and close post-operative follow-up with thyroglobulin. ${ }^{18)}$ Another study reported that the recurrence rate was $4 \%$ (range, $3-8 \%$ ) when the number of metastatic LN was less than five, and 19\% (range, 7-21\%) when the number was five or greater. ${ }^{14)}$ If there are metastatic LNs in a compartment, surgeons must perform comprehensive compartmental dissection and complete surgical removal of the metastatic LN. In this study, the presence of more than two metastatic LNs was significant in univariate analyses of recurrence or metastasis, but not in multivariate logistic regression analyses.

\section{Size of Metastatic LN Foci}

Previous studies have reported a micrometastasis incidence rate ranging from 25 to $65 \%$ in PTC patients. ${ }^{18,19)}$ Lang et al. ${ }^{18)}$ conducted a study on the size of metastatic $L N$ foci and reported that metastatic LN size affected post-operative stimulated thyroglobulin levels. Kim et al. ${ }^{20)}$ reported that macrometastasis was an independent prognostic factor for local recurrence, patients with macrometastases presented with significantly more lateral LN metastases. ${ }^{13)}$ These results indicate that PTMC patients with macrometastasis should be treated more aggressively and receive careful follow-up vigilance for recurrence. The 2015 American Thyroid Association Management Guidelines emphasized the importance of the number of $L N$ s examined and involved, the size of the largest metastatic LN focus, and ENE, and recommended standardized reporting of these items. In our study, micrometastasis was found in 76 of the $163(46.6 \%)$ study participants, 230 cases $(71.7 \%)$ represented the low-risk LN disease. Twenty of the 27 patients (74.1\%) with recurrence or metastasis were classified at high-risk LN disease, and this was significant in the univariate analysis, but not in the multivariate logistic regression analysis.

\section{LN Ratio}

Complete surgical removal of metastatic LNs may result in improved prognoses and a lower recurrence rate. $L N$ ratio is a prognostic variable in many cancers including thyroid, such as gastric, colorectal, and breast cancer. Using the number of metastatic LNs and the number of retrieved LNs, we can estimate the extent of nodal disease and assess how completely the LN was removed. The greater the number of metastatic LNs, the higher the LN ratio. Jeon et al. ${ }^{11)}$ reported that an $L N$ ratio of 0.4 or more was an important prognostic factor in pathological N1a, but this study did not find it to be significantly associated with recurrence or lateral LN metastasis through univariate and multivariate logistic regression analysis.

\section{Extranodal Extension}

ENE has a negative effect on disease-free and cause-specific survival and is associated with progression to high thyroglobulin, nodal persistence, and systemic disease. ${ }^{15,21)} \mathrm{Wu}$ et al. ${ }^{21)}$ found no difference in prognosis between $\mathrm{N1a}$ and $\mathrm{N} 1 \mathrm{~b}$ metastasis in the group without ENE. The prognosis of N1a patients with ENE was worse than in N1b patients. Some patients showed recurrence within six months after surgery, and it would be appropriate to classify this as persistent disease. These findings suggest that ENE is an important prognostic factor for survival and recurrence, and especially, it is the best predictor of death in PTC patients over 45 years of age without distant metastasis. ${ }^{21)}$ In our study, 10 PTC cases presented with concomitant ENE (3.1\%). This was uncommon, however, there were lateral LN metastases in six of these cases, observed in 2 out of the 4 cases with local LN recurrence in the central compartment. We found ENE was the most significant prognostic factor for recurrence or lateral LN metastasis (especially recurrence), with a hazard ratio of 10.3 .

Despite accumulating data, many institutions still do not report size and ENE of LN metastases. Characteristics of metastatic $L N$ are important for predicting risk factors for recurrence, deciding treatment options, and planning follow-up strategies. In this study, we dem- 
onstrated that LN characteristics do affect the likelihood of local recurrence or lateral LN metastasis in PTC patients. Patients classified as being at higher risk for recurrence and metastasis in postoperative stratification should be treated with postoperative RAI and TSH suppression to reduce this risk, and be carefully monitored with thyroglobulin, and receive regular US follow-ups to detect early recurrence.

In this study, long-term results could not be obtained because due to limitations on the short follow-up periods and the small number of patients presenting with a recurrence or metastasis. We recommend future studies with larger numbers of patients and longer-term follow-up to enable a more accurate assessment of risk factors for recurrence and metastasis and to help determine future treatment guidelines.

In conclusion, the multiple/bilateral cancer, ETE, metastatic LN size (macrometastasis), ENE, high-risk LN disease and high off-hormone thyroglobulin were associated with risk factors for metastasis or recurrence in PTC patients. The ENE was the most potent risk factors for central LN recurrence or lateral LN metastasis for PTC patients.

\section{Conflicts of Interest}

The authors report no conflict of interest.

\section{Orcid}

Wan Wook Kim: https://orcid.org/0000-0002-7363-5889 Jeeyeon Lee: https://orcid.org/0000-0003-1826-1690 Jin Hyang Jung: https://orcid.org/0000-0003-2607-1686 Ho Yong Park: https://orcid.org/0000-0002-4380-0089 Ji Yun Jeong: https://orcid.org/0000-0001-6167-4998 Ji-Young Park: https://orcid.org/0000-0002-7571-1064 Ralph P. Tufano: https://orcid.org/0000-0002-2219-3208

\section{References}

1) Seo GH, Chung JH. Analysis of therapeutic modalities of thyroid cancer in Korea between 2008 and 2012. J Korean Thyroid Assoc 2014; 7(02):167-71.

2) Cho BY, Choi HS, Park YJ, Lim JA, Ahn HY, Lee EK, et al. Changes in the clinicopathological characteristics and outcomes of thyroid cancer in Korea over the past four decades. Thyroid 2013;23(7):797-804.

3) Ortiz S, Rodriguez JM, Soria T, Perez-Flores D, Pinero A, Moreno J, et al. Extrathyroid spread in papillary carcinoma of the thyroid: clinicopathological and prognostic study. Otolaryngol Head Neck Surg 2001;124(3):261-5.

4) Shah JP, Loree TR, Dharker D, Strong EW, Begg C, Vlamis V. Prognostic factors in differentiated carcinoma of the thyroid gland. Am J Surg 1992;164(6):658-61.

5) Teixeira G, Teixeira T, Gubert F, Chikota H, Tufano R. The incidence of central neck micrometastatic disease in patients with papillary thyroid cancer staged preoperatively and intraoperatively as NO. Surgery 2011;150(6):1161-7.

6) Park CH, Song CM, Ji YB, Pyo JY, Yi KJ, Song YS, et al. Significance of the extracapsular spread of metastatic lymph nodes in papillary thyroid carcinoma. Clin Exp Otorhinolaryngol 2015;8(3):289-94.

7) Liu FH, Kuo SF, Hsueh C, Chao TC, Lin JD. Postoperative recurrence of papillary thyroid carcinoma with lymph node metastasis. J Surg Oncol 2015;112(2):149-54.

8) Grogan RH, Kaplan SP, Cao H, Weiss RE, Degroot LJ, Simon CA, et al. A study of recurrence and death from papillary thyroid cancer with 27 years of median follow-up. Surgery 2013; 154(6):1436-46; discussion 46-7.

9) Wada N, Suganuma N, Nakayama H, Masudo K, Rino Y, Masuda M, et al. Microscopic regional lymph node status in papillary thyroid carcinoma with and without lymphadenopathy and its relation to outcomes. Langenbecks Arch Surg 2007; 392(4):417-22.

10) Lundgren CI, Hall P, Dickman PW, Zedenius J. Clinically significant prognostic factors for differentiated thyroid carcinoma: a population-based, nested case-control study. Cancer 2006; 106(3):524-31.

11) Jeon MJ, Yoon JH, Han JM, Yim JH, Hong SJ, Song DE, et al. The prognostic value of the metastatic lymph node ratio and maximal metastatic tumor size in pathological N1a papillary thyroid carcinoma. Eur J Endocrinol 2013;168(2):219-25.

12) Clain JB, Scherl S, Dos Reis L, Turk A, Wenig BM, Mehra $\mathrm{S}$, et al. Extrathyroidal extension predicts extranodal extension in patients with positive lymph nodes: an important association that may affect clinical management. Thyroid 2014;24(6):951-7.

13) Cho SY, Lee TH, Ku YH, Kim HI, Lee GH, Kim MJ. Central lymph node metastasis in papillary thyroid microcarcinoma can be stratified according to the number, the size of metastatic foci, and the presence of desmoplasia. Surgery 2015;157(1):111-8.

14) Randolph GW, Duh QY, Heller KS, LiVolsi VA, Mandel SJ, Steward DL, et al. The prognostic significance of nodal metastases from papillary thyroid carcinoma can be stratified based on the size and number of metastatic lymph nodes, as well as the presence of extranodal extension. Thyroid 2012;22(11):1144-52.

15) Lango M, Flieder D, Arrangoiz R, Veloski C, Yu JQ, Li T, et al. Extranodal extension of metastatic papillary thyroid carcinoma: correlation with biochemical endpoints, nodal persistence, and systemic disease progression. Thyroid 2013;23(9): 1099-105.

16) Haugen BR, Alexander EK, Bible KC, Doherty GM, Mandel SJ, Nikiforov YE, et al. 2015 American Thyroid Association 
management guidelines for adult patients with thyroid nodules and differentiated thyroid cancer: the American Thyroid Association guidelines task force on thyroid nodules and differentiated thyroid cancer. Thyroid 2016;26(1):1-133.

17) Lee J, Song Y, Soh EY. Prognostic significance of the number of metastatic lymph nodes to stratify the risk of recurrence. World J Surg 2014;38(4):858-62.

18) Lang BH, Tang AH, Wong KP, Shek TW, Wan KY, Lo CY. Significance of size of lymph node metastasis on postsurgical stimulated thyroglobulin levels after prophylactic unilateral central neck dissection in papillary thyroid carcinoma. Ann Surg
Oncol 2012;19(11):3472-8.

19) Cranshaw IM, Carnaille B. Micrometastases in thyroid cancer. An important finding? Surg Oncol 2008;17(3):253-8.

20) Kim SY, Kim BW, Pyo JY, Hong SW, Chang HS, Park CS. Macrometastasis in papillary thyroid cancer patients is associated with higher recurrence in lateral neck nodes. World J Surg 2018;42(1):123-9.

21) Wu MH, Shen WT, Gosnell J, Duh QY. Prognostic significance of extranodal extension of regional lymph node metastasis in papillary thyroid cancer. Head Neck 2015;37(9): 1336-43. 